\title{
Effect of Probiotic Supplementation along with Calorie Restriction on Metabolic Endotoxemia, Trimethylamine-N-Oxide, Inflammation, Metabolic Factors, and Gut Microbiota Profile in Coronary Artery Disease Patients: A Double Blind Placebo Controlled Randomized Clinical Trial
}

Jalal Moludi ( $\sim$ jmoludi@yahoo.com)

Kermanshah University of Medical Sciences https://orcid.org/0000-0002-8333-414X

Hossein Samadi Kafil

Tabriz University of Medical Sciences

Pouria Gholizadeh

Tabriz University of Medical Sciences

Mohammad Alizadeh

Tabriz University of Medical Sciences

\section{Research}

Keywords: Coronary Artery Disease, lipopolysaccharide, gut microbiota, Metabolic endotoxmia, Probiotic, Trimethylamine-N-oxide

Posted Date: April 9th, 2020

DOl: https://doi.org/10.21203/rs.3.rs-17854/v2

License: (c) (i) This work is licensed under a Creative Commons Attribution 4.0 International License.

Read Full License 
The authors have withdrawn this preprint from Research Square 